\title{
Fatores associados a competência de comunicação entre enfermeiros cirúrgicos*
}

Factors associated with communication competence among surgical nurses

Factores asociados con la competencia de comunicación entre las enfermeras quirúrgicas

\section{Laura Andrian Leal ${ }^{\mathrm{I}}$, Beatriz Regina da Silva ${ }^{\mathrm{II}}$, Lucieli Dias Pedreschi Chaves ${ }^{\mathrm{III}}$, Mirelle Inácio Soares ${ }^{\mathrm{IV}}$, Daniela Sarreta Ignáciov, Silvia Helena Henriques ${ }^{\mathrm{VI}}$}

\begin{abstract}
Resumo: Objetivo: identificar os fatores associados a comunicação como competência, entre os enfermeiros de centros cirúrgicos. Método: estudo exploratório, qualitativo. Participaram 43 enfermeiros de cinco instituições hospitalares privadas do interior de Minas Gerais. A coleta ocorreu em 2018 por meio de seis encontros de grupos focais. Os dados foram analisados por análise temática indutiva. Resultados: evidenciou-se que a competência de comunicação está associada a fatores, como: qualidade e segurança no cuidado, relacionamento na equipe de trabalho, formas de se comunicar e interagir; informatização da passagem de plantão e interação com outros setores da instituição. Conclusão: considerando a comunicação como competência essencial ao enfermeiro cirúrgico, identificar fatores que possam influenciá-la de forma positiva ou limitante deve provocar a reflexão de futuros profissionais e gestores na elaboração e implantação de estratégias, no ambiente laboral, que promovam o desenvolvimento de uma comunicação eficaz.

Descritores: Comunicação; Competência profissional; Centros cirúrgicos; Enfermagem; Enfermagem perioperatória
\end{abstract}

\begin{abstract}
Objective: to identify the factors associated with communication as competence among nurses in surgical centers. Method: exploratory, qualitative study. The participants were 43 nurses from five private hospital institutions in the countryside of Minas Gerais participated. The collection occurred in 2018 through six meetings of focus groups. The data were analyzed by inductive thematic analysis. Results: communication competence is associated with factors such as: care quality and safety, relationship in the work team, ways of communicating and interacting; computerization of the shift and interaction with other sectors of the institution. Conclusion: considering communication as an essential competence for surgical nurses, identifying factors that can influence it in a positive or limiting way should provoke the
\end{abstract}

\footnotetext{
${ }^{I}$ Enfermeira, Doutoranda do Programa Interunidades da Escola de Enfermagem de Ribeirão Preto, Universidade de São Paulo. Ribeirão Preto, São Paulo, Brasil. laura.andrian.leal@usp.br Orcid: https://orcid.org/0000-0002-8563-8980.

II Enfermeira, Doutora em Ciências, Docente permanente da Faculdade Pitágoras. Uberlândia, Minas Gerais, Brasil. reginagava@yahoo.com.br. Orcid: https://orcid.org/0000-0001-5635-5757.

III Enfermeira, Doutora e Professora Associada da Escola de Enfermagem de Ribeirão Preto, Universidade de São Paulo. Ribeirão Preto, São Paulo, Brasil.dpchaves@eerp.usp.br Orcid: https://orcid.org/0000-0002-8730-2815.

IV Enfermeira. Docente titular do Centro Universitário de Lavras-UNILAVRAS. Lavras, Minas Gerais, Brasil. mirelleenfermagem@gmail.com Orcid: https://orcid.org/0000-0002-5298-8634.

V Enfermeira, Doutoranda do Programa de Enfermagem Fundamental da Escola de Enfermagem de Ribeirão Preto, Universidade de São Paulo. Ribeirão Preto, São Paulo, Brasil. daniela.sarreta@usp.br Orcid: https://orcid.org/0000-0002-1432-5098.

VI Enfermeira, Doutora e Professora Associada da Escola de Enfermagem de Ribeirão Preto, Universidade de São Paulo. shcamelo@eerp.usp.br Orcid: https://orcid.org/0000-0003-2089-3304.

* Extraído da tese "Matriz de Competências do enfermeiro que atua em Unidade de Centro Cirúrgico", apresentada ao Programa de Pós-Graduação Interunidades de Doutoramento em enfermagem, da Universidade de São Paulo, Escola de Enfermagem de Ribeirão Preto, em 2019.
} 
Fatores associados a competência de comunicação entre enfermeiros cirúrgicos $\mid 2$

reflection of future professionals and managers in the development and implementation of strategies in the work environment that promote the development of effective communication.

Descriptors: Communication; Professional Competence; Surgical centers; Nursing; Perioperative nursing

Resumen: Objetivo: identificar los factores asociados a la comunicación como competencia entre las enfermeras de los centros quirúrgicos. Método: estudio exploratorio y cualitativo. Participaron 43 tres enfermeras de cinco instituciones hospitalarias privadas del interior de Minas Gerais. La recogida se produjo en 2018 a través de seis reuniones de grupos focales. Los datos fueron analizados mediante análisis temáticos inductivos. Resultados: se evidenció que la competencia en comunicación está asociada a factores tales como: calidad y seguridad en la atención, relación en el equipo de trabajo, formas de comunicarse e interactuar; informatización del cambio e interacción con otros sectores de la institución. Conclusión: considerando la comunicación como una competencia esencial para las enfermeras quirúrgicas, identificar factores que puedan influir en ella de una manera positiva o limitante debe provocar la reflexión de futuros profesionales y gerentes en el desarrollo e implementación de estrategias en el entorno laboral que promuevan el desarrollo de una comunicación eficaz.

Descriptores: Comunicación; Competencia Profesional; Centros Quirúrgicos; Enfermería; Enfermería perioperatoria

\section{Introdução}

Em uma instituição hospitalar, a Unidade de Centro Cirúrgico (UCC) constitui-se pelo bloco cirúrgico, envolvendo suas salas operatórias e central de recuperação, que são dotadas por um ambiente complexo e estressante, munido de alta tecnologia, da variação intrínseca nos seus processos de trabalho e da situação de vulnerabilidade dos usuários. ${ }^{1}$ Ainda, nesta área hospitalar, pode-se destacar a multiplicidade de ações dos enfermeiros, que estão direcionadas ao planejamento do cuidado de pacientes cirúrgicos e de seus familiares, tanto na realização de ações assistenciais, como as gerenciais, relativas à organização do processo assistencial. ${ }^{2}$ Nessa direção, a comunicação é imprescindível para o processo de trabalho fluir de maneira assertiva e com menos riscos à segurança e integridade do usuário.

A comunicação pode ser conceituada como processo interpessoal entre pares, realizado de diversas formas, como verbal e não verbal nas interações, e que possui um impacto crucial na formação de normas sociais que, por sua vez, afetam o comportamento do indivíduo. ${ }^{3-4}$ Nessa linha de pensamento, a comunicação é uma competência descrita pela literatura como primordial para desenvolvimento de qualquer trabalho, ${ }^{5}$ e já está preconizado pelas Diretrizes Curriculares Nacionais (DCN) na formação do enfermeiro como ferramenta para prática. 
Entre essas diretivas cita-se a tomada de decisão, liderança, administração e gerenciamento, educação permanente e a comunicação. ${ }^{6}$ Entretanto, considera-se que esta se sobressai, uma vez que a prática dos enfermeiros está centrada na relação interpessoal com pacientes, equipe de enfermagem e equipe multiprofissional, tanto para realizar atividades assistenciais quanto para a gestão do cuidado e dos serviços de saúde. Além disso, ela perpassa e potencializa o desenvolvimento do trabalho e exercício das demais competências. ${ }^{7-8}$

Evidências científicas apontam diversas estratégias de ensino-aprendizagem sobre a comunicação, 5 ,9 no entanto, muitos enfermeiros ainda referem dificuldade em comunicar-se, especialmente em contextos que necessitam constantemente a tomada de decisão, como no caso de ambientes cirúrgicos. ${ }^{10}$ Contudo, apesar da gama de trabalhos publicados com a temática de desenvolvimento da comunicação, ressalta-se escassa literatura no que diz respeito a caracterização dos fatores associados.

Assim, apresentam-se as seguintes questões de pesquisa: Como é compreendida a competência da comunicação entre os enfermeiros das Unidades de Centros cirúrgico? Que fatores podem estar associados a ela?

Pensar na comunicação como competência ao enfermeiro de UCC, é refletir sobre as formas de receber, processar e transmitir respostas adequadas para usuários, equipe e gestores, contribuindo para evitar equívocos de mensagens e possibilitando melhorias nas relações interpessoais, gerando consequências diretas na qualidade do cuidado aos usuários. Além disso, a não identificação dos fatores que podem estar diretamente relacionados a esta competência profissional, para setores que tem especificidades como a UCC, pode acarretar problemas de relacionamentos e comportamentos na equipe, com consequências negativas para o cuidado prestado. Este estudo teve o objetivo de identificar os fatores associados a comunicação como competência, entre os enfermeiros de centros cirúrgicos. 


\section{Método}

Estudo exploratório, utilizando-se da abordagem qualitativa, aprovado pelo Comitê de Ética em Pesquisa da instituição proponente sob o parecer de número 087/2017 em 01 de fevereiro de 2017, e os participantes assinaram o Termo de Consentimento Livre e Esclarecido. O cenário foi constituído por cinco instituições hospitalares privadas de um município do Estado de Minas Gerais pelo fato de serem os locais que possuíam UCC próximos a sede residencial da pesquisadora. Os hospitais públicos deste local não aceitaram participar da pesquisa.

Os participantes foram compostos exclusivamente por enfermeiros assistenciais de UCC pertencentes aos hospitais selecionados, atuando há mais de seis meses na função. A escolha destes profissionais se refere ao fato de pertencerem a uma equipe de trabalho multiprofissional há algum tempo, de coordenar seu próprio pessoal, de realizar constantes troca de informações e, assim, ser capazes de descrever sobre o a competência de comunicação na UCC.

Primeiramente foi desenvolvido o contato com a enfermeira chefe de cada UCC que passou o número total de enfermeiros, e após estes foram formalmente e presencialmente convidados por meio de uma carta convite. Ademais, cinco enfermeiros que estavam em período de férias ou afastados por motivo de doença e licença maternidade foram excluídos da pesquisa. A população inicial era composta por 52 profissionais, entretanto nove se recusaram a participar sem um motivo aparente.

Os dados foram coletados no período de abril a junho de 2018, utilizando-se a técnica de Grupo Focal (GF), que garante ao pesquisador apreender informações in loco dos participantes da pesquisa por meio de colocações feitas pelo grupo. ${ }^{11}$ A realização do GF ocorreu nas próprias instituições de trabalho, em salas adequadas para discussão grupal, em horários de intervalos mais apropriado aos participantes, com duração média de 55 minutos e conduzidos pelo pesquisador (moderador) e um auxiliar de pesquisa (observador). O número de participantes em cada grupo foi 
5 | Leal LA, Silva BR, Chaves LDP, Soares MI, Ignácio DS, Henriques SH

organizado e distribuído conforme quadro abaixo (Quadro 1):

Quadro 1 - Distribuição do número de participantes em cada grupo focal realizado nas instituições hospitalares selecionadas. Brasil. 2019.

\begin{tabular}{|c|c|c|}
\hline Identificação do Hospital & Quantidade de GF realizados & $\begin{array}{c}\text { Número de enfermeiros } \\
\text { participantes em cada GF }\end{array}$ \\
\hline Hospital I & 1 & 8 \\
\hline Hospital II & 2 & 6 \\
\hline Hospital III & 1 & 6 \\
\hline Hospital IV & 1 & 7 \\
\hline Hospital V & 1 & 7 \\
\hline
\end{tabular}

Foram realizados dois grupos no Hospital II com enfermeiros diferentes, pelo fato de disponibilização de outros horários para que todos pudessem participar.

Ressalta-se que os grupos foram gravados e precedidos pelo preenchimento de um pequeno questionário para caracterização dos participantes e em seguida o moderador problematizou as discussões nos grupos focais por meio das questões norteadoras elencadas previamente. Os dados transcritos das discussões foram acrescidos às anotações e reflexões do moderador e do observador e submetidos a análise de conteúdo indutiva, ${ }^{12}$ que identifica, analisa e relata os padrões (temas) quanto aos dados; organiza e descreve seu conjunto em detalhes. Por meio de sua liberdade teórica, a análise temática proporciona uma ferramenta de pesquisa útil e flexível que pode potencialmente fornecer um relato rico, detalhado e complexo. ${ }^{12}$

Ressalta-se ainda que houve seguimento das etapas: transcrição e leitura dos dados; codificação; busca por temas por meio de códigos de agrupamento; revisando temas; análise em curso para aperfeiçoar as especificidades de cada tema; e, por fim, análise final dos trechos selecionados. ${ }^{12}$ Optou-se por utilizar a letra "E" de enfermeiro a fim de preservar o anonimato, seguida da identificação do hospital de onde foi realizado o estudo, de acordo com a ordem cronológica crescente da realização dos grupos de cada hospital (Exemplo: E7 - Hospital I). Ressalta-se ainda que para o hospital II, onde foram realizados dois grupos focais, foi identificado 
Fatores associados a competência de comunicação entre enfermeiros cirúrgicos |6

se era o grupo um ou dois (Exemplo: E1 - Hospital II grupo focal 1).

\section{Resultados}

Participaram do estudo 43 enfermeiros e a descrição sociodemográfica consta no Quadro 2:

Quadro 2- Dados sociodemográficos dos enfermeiros de Unidades de Centro Cirúrgicos do interior de Minas Gerais. Brasil. 2019.

\begin{tabular}{|c|c|c|c|}
\hline & & Frequência & Percentual \\
\hline \multirow[b]{2}{*}{ Sexo } & Feminino & 39 & 90,6 \\
\hline & Masculino & 4 & 9,4 \\
\hline \multirow{3}{*}{ Faixa etária } & 20-29 anos & 17 & 39,6 \\
\hline & 30-39 anos & 15 & 34,8 \\
\hline & $40-49$ anos & 11 & 25,6 \\
\hline \multirow{2}{*}{$\begin{array}{c}\text { Preparação durante } \\
\text { a Formação } \\
\text { acadêmica para } \\
\text { UCC }^{*}\end{array}$} & Sim & 30 & 69,7 \\
\hline & Não & 13 & 30,3 \\
\hline \multirow{2}{*}{$\begin{array}{c}\text { Especialização na } \\
\text { área de Centro } \\
\text { Cirúrgico }\end{array}$} & Sim & 7 & 16,3 \\
\hline & Não & 36 & 83,7 \\
\hline \multirow{2}{*}{$\begin{array}{l}\text { Especialização em } \\
\text { outras áreas }\end{array}$} & Sim & 10 & 23,3 \\
\hline & Não & 33 & 76,7 \\
\hline \multirow{6}{*}{$\begin{array}{c}\text { Tempo de atuação } \\
\text { na UCC* } \\
\text { selecionada }\end{array}$} & 1-2 anos & 9 & 20,9 \\
\hline & 3-5 anos & 8 & 18,6 \\
\hline & 6-7 anos & 8 & 18,6 \\
\hline & 7-8 anos & 8 & 18,6 \\
\hline & 9-10 anos & 5 & 11,6 \\
\hline & 10 ou mais anos & 5 & 11,6 \\
\hline
\end{tabular}

*Unidade de Centro Cirúrgico (UCC)

As especializações de outras áreas afins foram: Unidade de Terapia Intensiva (UTI) com ênfase em Urgência e Emergência, Auditoria, Gestão em Saúde e Controle de Infecção Hospitalar. Além disso 24 (58\%) afirmaram ter experiência profissional em UCC em outros hospitais com média de tempo de três anos de atuação. Os dados provenientes das gravações dos grupos evidenciaram que a competência de comunicação está associada com diversos fatores, distribuídos aqui em cinco categorias: "Qualidade e Segurança no Cuidado"; "Relacionamento interpessoal na equipe de trabalho"; "Formas de se comunicar e interagir nas UCC"; "Informatização da passagem de plantão" e "Interação com outros setores da Instituição". 


\section{Categoria 1: Qualidade e segurança no cuidado}

Os participantes reconhecem a importância da competência de comunicação para o processo de trabalho do enfermeiro em UCC, na garantia da qualidade e segurança do usuário, entretanto, nem sempre isso é realizado da forma ideal. Ademais a comunicação foi apontada como peça chave para utilização de outras competências que conferem qualidade na assistência, como liderança, planejamento e trabalho em equipe:

Eu acho que como todo enfermeiro bom hoje ele somente é se tiver uma comunicação efetiva porque tudo isso que as meninas falaram [planejamento, liderança, trabalho em equipe, gerenciamento da unidade] se isso tiver qualquer falha na comunicação vai ter algum impacto no processo como um todo. Sem essa comunicação de forma clara e de fato essa qualidade da informação tudo isso vai ficar perdido no processo como um todo. (E7 Hospital I)

[...] eu acho que a comunicação é uma competência muito importante que o enfermeiro deve ter para atuar em Centro Cirúrgico para segurança e qualidade diante de tantos equipamentos, apesar de nos comunicarmos, nem sempre fazemos uma comunicação efetiva e correta [...]. É, porque além de tudo, tem que voltar no que todo mundo falou que é trabalho em equipe, você trabalha com muita gente, saber se comunicar, entender o que as pessoas estão falando, o que elas querem de você naquele momento, eu acho que também é muito importante por isso precisa ter comunicação. (E5 - Hospital II grupo focal 2)

\section{Categoria 2: Relacionamento na equipe de trabalho}

Apesar dos participantes terem destacado que a comunicação está interligada com várias competências profissionais do enfermeiro, os grupos associaram mais fortemente como a comunicação pode interferir no relacionamento interpessoal entre as equipes e como pode amenizar conflitos internos:

A competência de comunicação está presente em todas as outras atividades e competências do enfermeiro aqui, mas eu acho que ela é mais presente ainda no relacionamento com os colegas [...]. Aqui tem muito conflito, e na maioria das vezes quando nós não conseguimos resolver com o médico aí nós 
Fatores associados a competência de comunicação entre enfermeiros cirúrgicos | 8

conversamos com a diretora clínica, então é assim se a nossa comunicação, nosso diálogo não deu certo nós conversamos com ela e aí ela como diretora conversa com eles ai ela resolve com eles. (E4 - Hospital II grupo focal 1)

Essa parte de comunicação com o relacionamento é a dificuldade, em lidar com os médicos [...]. Entrar aquela parte de jogo de cintura que nós temos que ter bastante, aquele jeito fácil de conversar com o médico, e técnicos. Já vem muito armados, então entra também a capacidade do enfermeiro de saber lidar muito bem com o médico dentro do Centro Cirúrgico, e é a comunicação que permite lidar com conflitos e relações. (E3 - Hospital V)

\section{Categoria 3: Formas de se comunicar e interagir nas UCC}

Para manter relacionamentos laborais saudáveis e segurança do processo de trabalho, os participantes revelaram algumas formas de comunicação utilizadas, como as formas verbais de maneira calma e firme, dispositivos virtuais, forma escrita como no prontuário, entre outras:

[...] eu acho que esse enfermeiro do setor ele pode se comunicar por meio de instrumentos que nós temos, seja verbal de maneira clara, calmo sem gritar, mas firme, seja por e-mail seja criação de qualquer processo. (E7 - Hospital I) [...] nós temos tem outras formas de comunicação além do WhatsApp E-mail, atas de reunião, lista de presença de treinamentos, relatório de não conformidade [...]. Nós temos um livro de passagem de plantão também que nós passamos o plantão pelo livro. [...] tudo anotado lá, além da Sistematização da Assistência de Enfermagem [...]. (E3 -Hospital I)

\section{Categoria 4: Informatização da passagem de plantão}

Nos discursos, os enfermeiros destacaram a utilização de sistema informatizado como meio de comunicação eficiente para passagem de plantão, possíveis de serem acessados em todos os setores da instituição. As informações inseridas nestes sistemas servem para levantamentos de dados, análise e supervisão de indicadores de qualidade hospitalar. Contudo, um grupo apontou que a comunicação na passagem de plantão era feita por contato via telefone com equipe médica e de enfermagem e passagem de plantão verbal.

Nós usamos um Sistema Estratégico de Ocorrências [...] daí fazemos essa comunicação e ela não é perdida, fica registrado; onde toda a equipe abre e 
que direciona para as pessoas responsáveis que serão capazes de resolver essa problemática relacionada a passagem de plantão, e aí passa isso e a equipe ela deve receber o retorno do que foi feito para corrigir a falhas então assim isso acontece na equipe hospitalar. (E2 - Hospital II grupo focal 1)

Aqui a gente não tem ainda sistema informatizado para passar plantão, nós falamos por telefone ou verbalmente mesmo. (E1- Hospital I)

\section{Categoria 5: Interação com outros setores da instituição}

Por fim, durante as discussões os participantes relataram diversas vivências com relação à falta de material, equipamento, necessidade de reparos nos aparelhos, falta de roupas, aventais cirúrgicos e campos cirúrgicos, que exigiam do enfermeiro a capacidade de se comunicar tanto com sua equipe, como com gestores ou até mesmo outras instituições por meio do diálogo, das parcerias e de uma boa convivência, visando o alcance das metas organizacionais. Nessa perspectiva, os enfermeiros consideram relevante estreitar os laços de comunicação entre os setores de apoio como Central de Material e Esterilização (CME), lavanderia e outros.

[...] questão do material não é só o agendamento de cirurgia, a gente tem de ter uma comunicação, o que vai precisar? Quando vai precisar? Porque a gente tem um tempo para esterilizar o material, receber ou lavar, processar algum instrumento, entendeu? Isso leva tempo. Então precisa ter comunicação entre os setores CME e UCC [...]. (E5 - Hospital III)

Com a CME temos comunicação o dia inteiro. Preciso do material, vou usar tal hora, agiliza ele aí; já dá, para o material, prioridade na autoclave; deixa a autoclave aberta que daqui a pouco vou descer. Então é o tempo inteiro, não chegou roupa, vai vendo o que vai dando para improvisar, usar outro tipo de campo?(E6 - Hospital III)

\section{Discussão}

O estudo revelou população predominantemente jovem, e com múltiplas especializações, corroborando com outros pesquisadores que evidenciaram a inserção de enfermeiros jovens em setores complexos de atendimento e, ainda identificaram dificuldades em estabelecer uma comunicação efetiva, que é aquela que um indivíduo sabe como filtrar e organizar as informações que deve transmitir e escolher a forma ideal de usar os canais para difundir seus pensamentos em 
Fatores associados a competência de comunicação entre enfermeiros cirúrgicos $\mid 10$ seu processo de trabalho. ${ }^{13-14}$ A variedade de especializações demonstra a preocupação e necessidade do profissional em qualificar seu trabalho, possivelmente atribuído a lacunas da formação acadêmica, bem como à complexidade do mercado de atuação.

Os enfermeiros das UCC(s) selecionadas reconheceram a relevância da utilização da comunicação para segurança e qualidade da assistência, entretanto é necessário refletir sobre o que é idealmente proposto e o que de fato é realizado acerca desta competência. Pesquisadores americanos e brasileiros constataram que enfermeiros não tem uma comunicação clara e efetiva durante a assistência hospitalar, que pode ser atribuído a falta de aprimoramentos oferecidos pela instituição empregadora, organizações com sistemas de hierarquias autoritárias e os diferenciais de poder associados à antiguidade e ao status profissional, além de profissionais jovens com pouca experiência. $^{13,15}$

Acredita-se que o fluxo de transmissão da informação por vários níveis hierárquicos e por diversos profissionais pode ocasionar comunicação ineficaz; por conseguinte, pode afetar a qualidade nos serviços prestados por esses profissionais e consequentemente a segurança do paciente. Nesse sentido, é válido dizer que a estrutura organizacional tem potencial para interferir de forma positiva ou negativa no processo de comunicação entre os seus membros constituintes. Vale destacar que a comunicação é um dos cinco principais problemas que afetam a segurança do paciente; por isso está relacionada a qualidade do cuidado, onde ações visando a melhoria no processo de emissão e transmissão de informações fazem parte das diretrizes mundiais para padronização e implantação de protocolos para a redução de eventos adversos, maior segurança e qualidade assistencial. ${ }^{16-17}$

Pesquisadores evidenciaram que o nível de cultura de segurança do paciente em UCC está abaixo do preconizado na literatura. ${ }^{18}$ Em relação a isso, apresentaram por meio da média geral de escores, uma cultura de segurança incipiente, e quando avaliada por domínios, satisfação do trabalho e percepção do estresse mostraram-se como positivos. Identificaram, ainda, que clima de trabalho em equipe, clima de segurança, percepção da gerência da unidade/hospital e condições de 
trabalho, foram indicados como domínios negativos, sendo sugeridas intervenções a fim de promover um ambiente seguro, como processos de educação continuada e introdução de novas ferramentas, como o checklist de segurança cirúrgica e comunicação sistematizada. ${ }^{18}$

A competência de comunicação também foi associada às interações com a equipe de trabalho, ou seja, com capacidade para interferir nas relações interpessoais entre os membros da equipe. Acredita-se que falhas na obtenção, no processamento e transmissão de informações, podem levar a insatisfação e estresse dos profissionais gerando conflitos internos. Desta forma, ambientes de trabalho onde possuem um constante diálogo e trocas de saberes possibilitam a amenização de conflitos e possivelmente um planejamento participativo coletivo que potencializam a integração dos diversos profissionais. ${ }^{19}$

No processo de trabalho gerencial do enfermeiro cirúrgico, a competência comunicativa é fundamental para que haja interações adequadas e produtivas; isso implica conhecer os elementos da comunicação, saber dialogar, conhecer profundamente a dinâmica de trabalho para encontrar brechas ou alternativas para identificar e solucionar as necessidades dos usuários, criando vínculo, agregando humanização ao cuidado.

Assim, o enfermeiro é reconhecido como o profissional que deve propiciar a comunicação entre os diversos trabalhadores da equipe de saúde, bem como com seus usuários, atendendo dessa forma, as necessidades dos que requerem solução compartilhada, contribuindo para o cuidado integral a saúde. ${ }^{20}$

Para garantia de interações saudáveis, a competência de comunicação pode ser realizada de diversas formas. A comunicação verbal diz respeito a linguagem e a escrita, bem como os sons das palavras emitidas. No que diz respeito a comunicação não verbal, esta se refere a utilização de gestos, toque, postura, expressões, objetos que cercam uma pessoa ou posição perante o outro. ${ }^{21}$

Além disso, o uso de mídias sociais e aplicativos, a exemplo, o WhatsApp, já vêm sendo utilizados no meio acadêmico na área da enfermagem, como aponta alguns estudos, para facilitar a comunicação, a troca de informações e o aprendizado das práticas em saúde. ${ }^{22-23}$ Essas ferramentas 
Fatores associados a competência de comunicação entre enfermeiros cirúrgicos | 12

têm como vantagens o compartilhamento de conteúdo para o desenvolvimento de discussões de diversos temas, possibilidade de expor suas opiniões e compartilhar os conhecimentos e a experiência clínica. ${ }^{24}$ Deste modo, observa-se cada vez mais a incorporação de ferramentas tecnológicas para desenvolvimento de comunicação efetiva no ambiente hospitalar, e outro exemplo é a utilização de sistemas informatizados para passagem de plantão, o que foi apontado em nosso estudo.

A utilização de recursos informatizados está ganhando cada vez mais espaço nas instituições hospitalares e, representam ferramentas para auxiliar a transmissão de dados durante a passagem de plantão; favorecendo que os registros permaneçam gravados e possam ser acessados a qualquer momento, agilizando assim a transferência das informações e repercutindo na observância da jornada de trabalho. ${ }^{25-26}$

Entretanto, não são todas as instituições que possuem recursos financeiros disponíveis para arcar com essas tecnologias, assim estimula-se, dentre os recursos utilizados para a passagem de plantão, a interação entre a equipe de enfermagem que está finalizando o turno e a que inicia o plantão subsequente, ilustrando-se, com recursos adicionais da escrita, que podem ser facilmente acessadas posteriormente, como em lousas, censos em papel e slides. ${ }^{26}$

Destarte, as inovações tecnológicas são realidades do processo de trabalho da enfermagem, sendo importante refletir sobre o desafio de acompanhar a tecnologia sem negligenciar os aspectos humanitários inerentes a profissão. Nessa linha, há evidências que apontam benefícios da informatização, sendo que o cuidado deve ser assessorado por equipamentos, mas conduzido por pessoas ao articular humanização do cuidado à evolução da tecnologia e da ciência. ${ }^{27}$ Finalmente, para que possa dimensionar as cirurgias, bem como os cuidados com os pacientes submetidos a procedimentos cirúrgicos é preciso ter comunicação diária com diversas unidades de diferentes áreas/setores. ${ }^{28}$

Nessa direção, a UCC não funciona sozinha, e tem vários setores de apoio às suas atividades, a exemplo: CME, manutenção, farmácia satélite, almoxarifado, copa para os funcionários, serviço de 
limpeza, lavanderia que recolhe as roupas sujas, área de transporte e internação, repartições como as unidades de tratamento intensivo, e outros, essenciais para o funcionamento adequado deste campo. Compreende-se que a comunicação participativa entre profissionais de saúde e gestores, com a ajuda de ferramentas de integração, torna-se essencial para o bom andamento dos processos da instituição.

Nesse sentido, a utilização de estratégias de ensino que possam potencializar o desenvolvimento de competências de comunicação em enfermagem se faz essencial, visto que os estudantes ingressam cada vez mais jovens no ensino superior. Entre as ferramentas que podem ser aplicadas, destaca-se o potencial da simulação clínica, que possibilita aos discentes a vivência de situações que exigem tomada de decisão do enfermeiro nos cenários de cuidado em saúde e enfermagem; documentos formalizados de passagem de plantão ou de avisos/informes; canais de comunicação; reuniões e grupos de discussão multidisciplinar. ${ }^{5}$ Ademais espera-se que os centros formadores devam se preocupar em elaborar e/ou implementar em seus currículos metodologias ativas de aprendizagem para os futuros profissionais, visando contribuir para o seu conhecimento dessa competência. ${ }^{5,29-30}$

A investigação apresentou a limitação de ter sido realizado somente em hospitais privados e com participação exclusiva de enfermeiros; ao considerar que o trabalho em equipe multiprofissional é uma característica das UCC, as participações de outras categorias profissionais em pesquisas semelhantes podem trazer contribuições para futuros enfermeiros desta área.

\section{Conclusão}

O estudo permitiu identificar os fatores associados a comunicação como competência, entre os enfermeiros de centros cirúrgicos. Em todo processo de trabalho que envolve interações humanas o desenvolvimento adequado desta competência se faz essencial. A análise permitiu associar este fator à qualidade e segurança no cuidado, relacionamento interpessoal na equipe de trabalho, formas de se comunicar, sistema informatizado para passagem e plantão e interação com 
Fatores associados a competência de comunicação entre enfermeiros cirúrgicos | 14

outros setores da organização.

Sabe-se que ter uma comunicação efetiva dentro de uma instituição de saúde representa mais do que ter boas conversas e um bom relacionamento interpessoal com colegas e superiores, ou ainda de saber fazer relatórios escritos e mandar e-mails. A interlocução, neste contexto, está relacionada a benefícios, que se mostram na forma como os profissionais se comunicam com os clientes, colegas, líderes. Deste modo, além de ajudar na harmonização do ambiente, o desenvolvimento de um diálogo efetivo também traz excelentes resultados de curto, médio e longo prazo à organização, e aos usuários.

A realização de pesquisas que possam caracterizar e fundamentar o desenvolvimento da competência de comunicação em enfermeiros são primordiais, pois possibilita identificar lacunas existentes no que diz respeito a formação do profissional, além de suscitar gestores na implementação de estratégias que possam aprimorar seus trabalhadores, a exemplo, mediante integração com centros formadores. Ainda, este estudo pode contribuir sobremaneira para UCC, pois possibilita a reflexão dos profissionais que ali atuam, sobre as implicâncias do uso ineficaz da comunicação, com repercussões nas condições de recuperação dos indivíduos. Ainda, sugere-se a realização de outras pesquisas sobre a temática em diferentes cenários e sob a ótica de diferentes profissionais de saúde, incluindo instituições hospitalares de natureza jurídica diversa.

\section{Referências}

1. Appelbaum NP, Lee N, Amendola M, Dodson K, Kaplan B. Surgical resident burnout and job satisfaction: the role of workplace climate and perceived support. J Surg Res. 2019;234:20-5. doi: 10.1016/j.jss.2018.08.035

2. Pinno C, Camponogara S, Beck CLC. The dramatic "use of self' in the work of the nursing team in the surgical in-patient unit. Texto Contexto Enferm. 2019;28:e20170576. doi: 10.1590/1980-265x-tce-2017-0576

3. Geber S, Baumann E, Klimmt C. Where do norms come from? Peer communication as a factor in normative social influences on risk behavior. Commun Res. 2017;46(5):708-30. doi: 10.1177/0093650217718656

4. Hellmann A, Ang L, Sood S. Towards a conceptual framework for analysing impression management during face-to-face communication. J Behav Experiment Finance. 2020;25(e100265):1-4. doi: 10.1016/j.jbef.2020.100265

5. Soares MI, Silva BR, Leal LA, Brito LJS, Resck ZMR, Henriques SH. Strategies for the development of communication in an urgency and emergency hospital. REME Rev Min Enferm. 2020;24:e-1308. doi: 


\section{$10.5935 / 1415-2762.20200045$}

6. BRASIL. Ministério da Educação. Resolução CNE/CES n 3, de 7 de novembro de 2001. Institui Diretrizes Curriculares Nacionais do Curso de Graduação em Enfermagem. Diário Oficial da União: seção 1, Brasília, DF, n. 215, p. 37, 9 nov. 2001.

7. Lopes RCC, Azeredo ZAS, Rodrigues RMC. Relational skills of nursing students: follow-up of an intervention program. Referência. 2013;serIII(9):27-36. doi: 10.12707/RIII1253

8. Vasconcelos RMA, Caldana G, Lima EC, Silva LDM, Bernardes A, Silvia CG. Communication in the relationship between leaders and lead in the context of nursing. Rev Enferm UFPE [Internet]. 2017 [cited 2020 Oct 08];11(Supl 11):4767-6. Available from: https://periodicos.ufpe.br/revistas/revistaenfermagem/article/view/231220

9. Dalcól C, Garanhani ML, Fonseca LF, Carvalho BG. Communication skills and teaching-learning strategies: perception of nursing students. Cogitare Enferm. 2018;23(3):e53743. doi: 10.5380/ce.v23i3.53743

10. Banerjee SC, Manna R, Coyle N, Penn S, Gallegos TE, Zaider T, et al. The implementation and evaluation of a communication skills training program for oncology nurses. Translat Behav Med. 2017;7(3):615-23. doi: 10.1007/s13142-017-0473-5

11. Silva MG, Fernandes JD, Rebouças LC, Rodrigues GRS, Teixeira GA, Silva RMO. Publications that used focal group as research technique: what do they teach us? Ciênc Cuid Saúde. 2013;12(2):398-406. doi: 10.4025/cienccuidsaude.v12i2.9194

12. Braun V, Clarke V. Using thematic analysis in psychology. Qual Res Psychol. 2006;3(2):77-101. doi: 10.1191/1478088706qp063oa

13. Omura M, Stone TE, Maguire J, Levett-Jones T. Exploring Japanese nurses' perceptions of the relevance and use of assertive communication in healthcare: a qualitative study informed by the Theory of Planned Behaviour. Nurse Educ Today. 2018;67:100-7. doi: 10.1016/j.nedt.2018.05.004

14. Almeida RO, Ferreira MA, Silva RC. O cuidado intensivo em unidades não-críticas: representações e práticas de enfermeiros recém-formados. Texto Contexto Enferm. 2020;29:e20190089. doi: 10.1590/1980-265xtce-2019-0089

15. Soares MI, Leal LA, Resck ZMR, Terra FS, Chaves LDP, Henriques SH. Competence-based performance evaluation in hospital nurses. Rev Latinoam Enferm. 2019;27:e3184. doi: 10.1590/1518-8345.3173.3184

16. World Health Organization (WHO). The High 5s Project: interim report [Internet]. Geneva: World Health Organization; 2014 [cited 2020 Oct 05]. Avaliable from: http://www.who.int/patientsafety/implementation/solutions/high5s/High5_InterimReport.pdf

17. Souza ATG, Silva TKP, Domingues AN, Tognoli SH, Eduardo AHA, Macedo JI, et al. Segurança do paciente em centro cirúrgico: percepção dos profissionais de enfermagem. Rev SOBECC [Internet]. 2020 [acesso 2020 out 08];25(2):75-82. Disponível em: https://revista.sobecc.org.br/sobecc/article/view/593/pdf\#

18. Silva Júnior JF, Jesus Júnior PJ, Carvalho TA, Aguiar MPC, Mendonça SCB, Lordelo DS. Cultura de segurança do paciente: percepções e atitudes dos trabalhadores de centro cirúrgico. Rev SOBECC [Internet]. 2020 [acesso

2020 out 08];25(3):136-142. Disponível em: 
Fatores associados a competência de comunicação entre enfermeiros cirúrgicos | 16

https://pesquisa.bvsalud.org/controlecancer/resource/pt/biblio-1122488?src=similardocs

19. Martins FZ, Dall'agnol CM. Surgical center: challenges and strategies for nurses in managerial activities. Rev Gaúcha Enferm. 2016;37(4):1-9. doi: 10.1590/1983-1447.2016.04.56945

20. Negreiros FDS, Pequeno AMC, Garcia JHP, Aguiar MIF, Moreira TR, Flor MJN. Multi-professional team's perception of nurses' competences in liver transplantations. Rev Bras Enferm [Internet]. 2017 [cited 2020 Oct 08];70(2):242-8. Available from: https:/www.scielo.br/scielo.php?pid=S003471672017000200242\&script=sci_arttext

21. Broca PV, Ferreira MA. Communication process in the nursing team based on the dialogue between Berlo and King. Esc Anna Nery Rev Enferm. 2015;19(3):467-74. doi: 10.5935/1414-8145.20150062

22. Willemse JJ. Undergraduate nurses reflections on Whatsapp use in improving primary health care education. Curationis. 2015;38(2):a1512. doi: 10.4102/curationis.v38i2.1512

23. Bardaquim VA, Santos SVM, Bertolini SRF, Silva AF, Robazzi MLCC. The use of the application "whatsapp": facilitator of the communication in graduation discipline in nursing. Rev Teo Prat Educ. 2018;18(1):15-9.

24. Mesquita AC, Zamarioli CM, Fulquini FL, Carvalho EC, Angerami ELS. Social networks in nursing work processes: an integrative literature review. Rev Esc Enferm USP. 2017;51:e03219. doi: 10.1590/S1980220X2016021603219

25. Robinson AS, Baker SD, Smith BT, Agdeppa ED, Wells P, Hassey LA, et al. System and method for caregiver shift change. US Pat Appl Publ [Internet]. 2019 [cited 2020 Oct 08];14:1-15. Available from: https://patentimages.storage.googleapis.com/4d/57/eb/350996f6acbe10/US20200090804A1.pdf

26. Peruzzi LM, Goulart BF, Henriques SH, Alves LR, Laus AM, Chaves LDP. Switching shifts in hospital care. Rev Enferm UFPE On Line [Internet]. 2019 [cited 2020 Oct 08];13(4):989-6. Available from: https://periodicos.ufpe.br/revistas/revistaenfermagem/article/view/236967

27. Donoso MTV, Souza MAF, Mattos SS, Campos DMP, Silqueira SMF, Sharry S. Nursing in intensive care units: the technological apparatus versus care humanization. Rev Enferm Cent-Oeste Min. 2017;7:e1883. doi: 10.19175/recom.v7i0.1883

28. Gomes LC, Dutra KE, Pereira ALS. O enfermeiro no gerenciamento do centro cirúrgico. Rev Eletrônica Fac Metodista Granbery [Internet]. 2014 [acesso 2020 Out 08];16:1-21. Disponível em: http://re.granbery.edu.br/artigos/NTEy.pdf

29. Sánchez Expósito J, Leal Costa C, Díaz Agea JL, Carrilo Izquierdo MD, Jiménez Rodríguez D. Socioemotional competencies as predictors of performance of nursing students in simulated clinical practice. Nurse Educ Pract. 2018;32:122-8. doi: 10.1016/j.nepr.2018.07.009

30. Mukamana D, Karonkano GR, Rosa W. Advancing perioperative nursing in Rwanda through global partnerships and collaboration. Aorn [Internet]. 2016 [cited 2020 Oct 08];104(6):583-7. Available from: https://aornjournal.onlinelibrary.wiley.com/doi/full/10.1016/j.aorn.2016.10.006

Editora Científica Chefe: Cristiane Cardoso de Paula 


\section{7 | Leal LA, Silva BR, Chaves LDP, Soares MI, Ignácio DS, Henriques SH}

\section{Editora associada: Rosângela Marion da Silva}

Fomento: Financiamento Próprio.

\section{Autor correspondente}

Laura Andrian Leal

E-mail: laura.andrian.leal@usp.br

Endereço: Avenida Bandeirantes 3900, Escola de Enfermagem de Ribeirão Preto. Ribeirão Preto-SP. CEP: $14040-902$.

\section{Contribuições de Autoria}

\section{1 - Laura Andrian Leal}

Concepção e projeto, coleta, análise, interpretação dos dados e participação ativa na discussão dos resultados; Redação do artigo ou revisão crítica relevante do conteúdo intelectual; Revisão e aprovação final da versão a ser publicada e Concordância com todos os aspectos do manuscrito em termos de veracidade ou integridade das informações.

\section{2 - Beatriz Regina da Silva}

Concepção e projeto, coleta, análise, interpretação dos dados e participação ativa na discussão dos resultados; Redação do artigo ou revisão crítica relevante do conteúdo intelectual; Revisão e aprovação final da versão a ser publicada e Concordância com todos os aspectos do manuscrito em termos de veracidade ou integridade das informações.

\section{3 - Lucieli Dias Pedreschi Chaves}

Redação do artigo ou revisão crítica relevante do conteúdo intelectual; Revisão e aprovação final da versão a ser publicada e Concordância com todos os aspectos do manuscrito em termos de veracidade ou integridade das informações.

\section{4 - Mirelle Inácio Soares}

Redação do artigo ou revisão crítica relevante do conteúdo intelectual; Revisão e aprovação final da versão a ser publicada e Concordância com todos os aspectos do manuscrito em termos de veracidade ou integridade das informações.

\section{5 - Daniela Sarreta Ignácio}

Redação do artigo ou revisão crítica relevante do conteúdo intelectual; Revisão e aprovação final da versão a ser publicada e Concordância com todos os aspectos do manuscrito em termos de veracidade ou integridade das informações.

\section{6 - Silvia Helena Henriques}

Concepção e projeto, coleta, análise, interpretação dos dados e participação ativa na discussão dos resultados; Redação do artigo ou revisão crítica relevante do conteúdo intelectual; Revisão e aprovação final da versão a ser publicada e Concordância com todos os aspectos do manuscrito em termos de veracidade ou integridade das informações.

\section{Como citar este artigo}

Leal LA, Silva BR, Chaves LDP, Soares MI, Ignácio DS, Henriques SH. Factors associated with communication competence among surgical nurses. Rev. Enferm. UFSM. 2021 [Cited: Year Month Day]; vol.11 e51: 1-17. DOI: https://doi.org/10.5902/2179769261373 